\begin{tabular}{|l|l|l|}
\hline $\begin{array}{l}080 \text { - } 081 \\
\text { Debate } \\
\text { Proyectos y experiencias } \\
\text { PH46-Diciembre 2003 }\end{array}$ & $\begin{array}{l}\text { El Sistema de Información del Patrimonio Arquitectónico } \\
\text { (IPA) aplicado a las ciudades históricas de Portugal }\end{array}$ \\
\hline
\end{tabular}

Cuando, en 1964, los técnicos responsables de la salvaguardia de los monumentos históricos firmaran la Carta de Venecia, fueron elaborados otros doce documentos, el octavo de los cuales hacía referencia a la protección y rehabilitación de los centros históricos.

Aunque el primer documento se ha convertido en el texto de referencia de los profesionales del área, los principios y objetivos de éste último sólo dos décadas más tarde se consagrarian como texto definitivo, con la designación de Carta Internacional para la Salvaguardia de las Ciudades Históricas.

Resalta del preámbulo de este documento la ampliación que el concepto de patrimonio venía registrando, traducido en el párrafo inicial del texto definitivo: "Como resultado de un desarrollo espontáneo o de un proyecto deliberado, todas las ciudades del mundo son expresiones materiales de la diversidad de las sociedades a través de la historia, considerándose por ello todas históricas".

Este nuevo concepto del patrimonio, enriquecido por el valor documental que en- cierra, implica el reconocimiento no sólo de la importancia histórica y arquitectónica de los edificios, sino también, de las estructuras sociales que lo soportan.

Cada ciudad tiene un patrimonio y un conjunto de características tales que la convierten en única, dependiendo su conservación del modo en que la población los interpreta, los utiliza y los potencia en la regeneración de la ciudad. Estas caracteristicas, frágiles y perecederas, constituyen un factor de identidad colectiva y un motor de desarrollo económico y social de las comunidades. La necesidad de concienciación de los técnicos y gestores municipales, en lo relativo al impacto social y económico del patrimonio, vino a ser tema del encuentro de especialistas "Desarrollo e Integridad de las Ciudades Históricas", realizado en Nara en mayo de 1999. En este encuentro fue nuevamente subrayado que las ciudades están constituidas por un conjunto de elementos físicos -edificios, calles, plazas y jardines- $y$, también por actividades humanas y bienes intangibles que, conjuntamente,

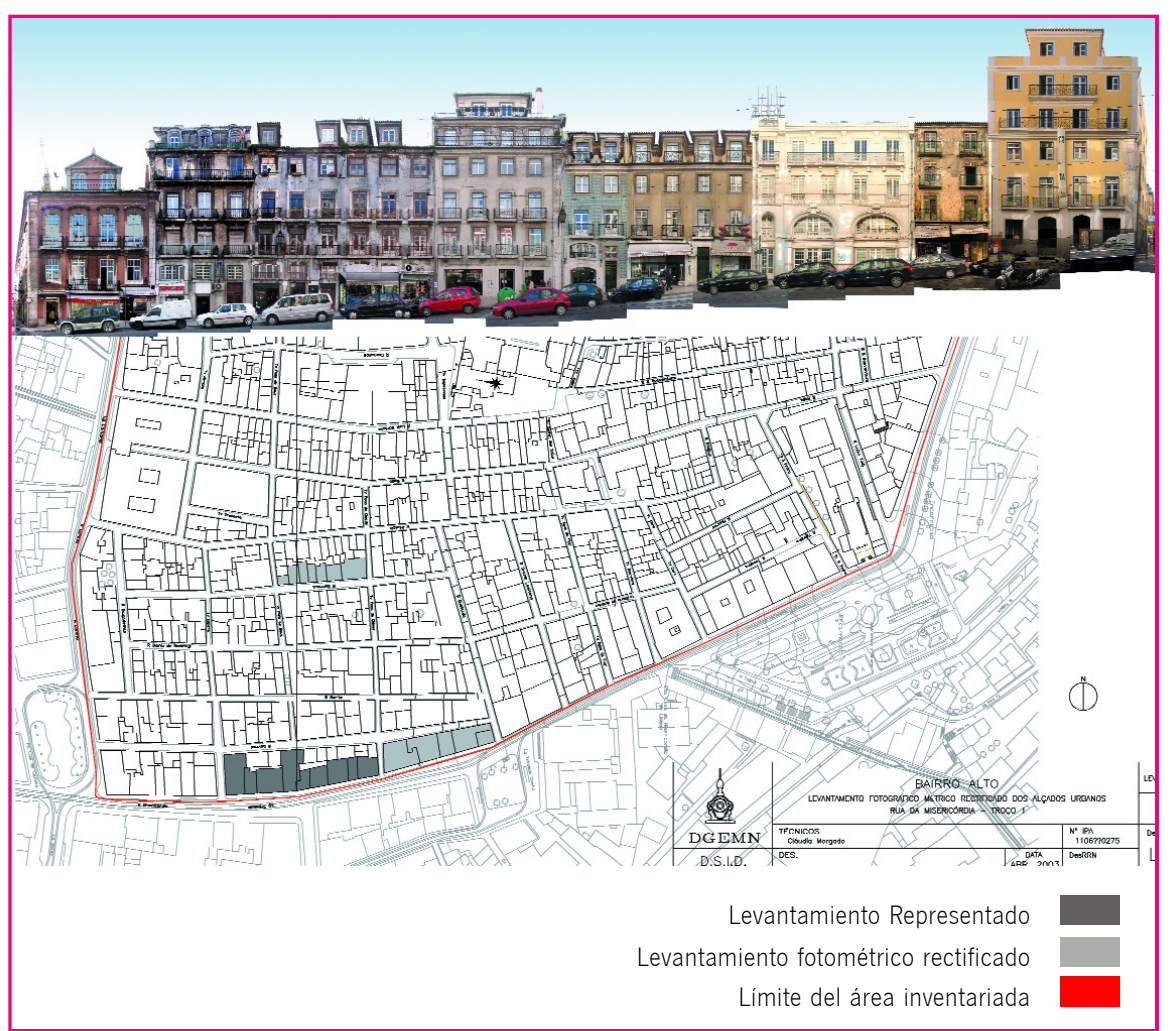

Lisboa. Barrio Alto

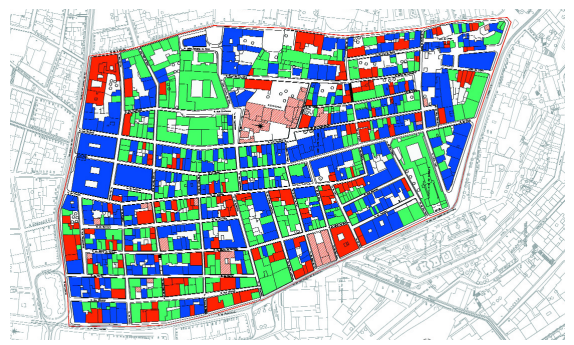

Lisboa. Barrio Alto, planta

sedimentan a lo largo de los tiempos su individualidad.

Siendo por todos reconocido que el valor de la ciudad depende del modo en el que su individualidad es comprendida, valorizada y mantenida, se hace urgente la implementación de medidas que aseguren la regeneración sostenible de las ciudades y su futuro a largo plazo.

Es en este contexto en el que la DGEMN desarrolló a partir del inicio de la década de los 90 el proyecto de creación de un Sistema de Información para el Patrimonio Arquitectónico (IPA), un instrumento de registro sistemático y en permanente actualización de datos textuales e iconográficos, de naturaleza técnica, científica y administrativa, sobre el universo del patrimonio arquitectónico portugués.

Este sistema, articulado en un conjunto de bases de datos interrelacionadas se constituye como un SIG (sistema de información geográfico), pudiendo relacionarse con otras bases de datos georeferenciadas, desarrolladas con el objetivo de conjugar y optimizar recursos esenciales para las tareas de planeamiento urbano y desarrollo económico de las regiones.

Después de haber inventariado un número representativo de inmuebles de mayor valor patrimonial, procuramos desarrollar una metodología que nos asegurase la necesaria/suficiente fiabilidad y mucho mayor rapidez en la recogida de la información indispensable para la caracterización de un núcleo urbano y para las acciones de protección, rehabilitación y planeamiento.

Esta metodología vino a dar origen a una de las bases de datos que constituyen el siste- 
ma IPA, la base de datos de los Conjuntos Urbanos que tiene por finalidad asegurar el conocimiento, la comprensión y gestión del tejido urbano, registrando y analizando las caracteristicas propias de cada ciudad y garantizando la información de base necesaria para la toma de decisión sobre programas o proyectos de intervención que respeten la diversidad regional y local.

Este proyecto pretende, de forma sistemática, recoger y registrar la información sobre patrimonio arquitectónico entendido como un todo construido, teniendo como objetivo constituir un recurso de información de apoyo para las acciones de salvaguardia y valorización de ese patrimonio, ya sean por iniciativa de la DGEMN o de otras entidades.

Esta metodología nos permitió transformar el IPA en un instrumento de gestión y comprensión del tejido urbano como un todo orgánico y de las características a la cualidad única de cada ciudad.

La información georeferenciada a cada área es registrada en una base de datos cuya actualización sistemática posibilita la producción de una cartografía temática: toponimia, trazado urbano, jerarquía viaria, tipología de lo edificado, utilización de inmuebles, evolución urbana, estado de conservación, adulteraciones registradas, entre otras. La cartografía temática es complementada por documentación fotográfica métrica que, tras rectificación, permite realizar alzados urbanos pasivos que serán tratados con CAD, siempre que sea necesario, para el desarrollo de propuestas de intervención y planos de salvaguardia.

Con la utilización de la fotografía métrica rectificada obtenemos documentación técnica de gran precisión, valor informativo y rapidez de ejecución, para:

> Registrar la silueta del estructura y la posición relativa del inmueble

$>$ Documentar los materiales, colores y estado de conservación de las fachadas

$>$ Registrar los pormenores arquitectónicos y constructivos a tener en cuenta en futuras intervenciones de rehabilitación de los inmuebles

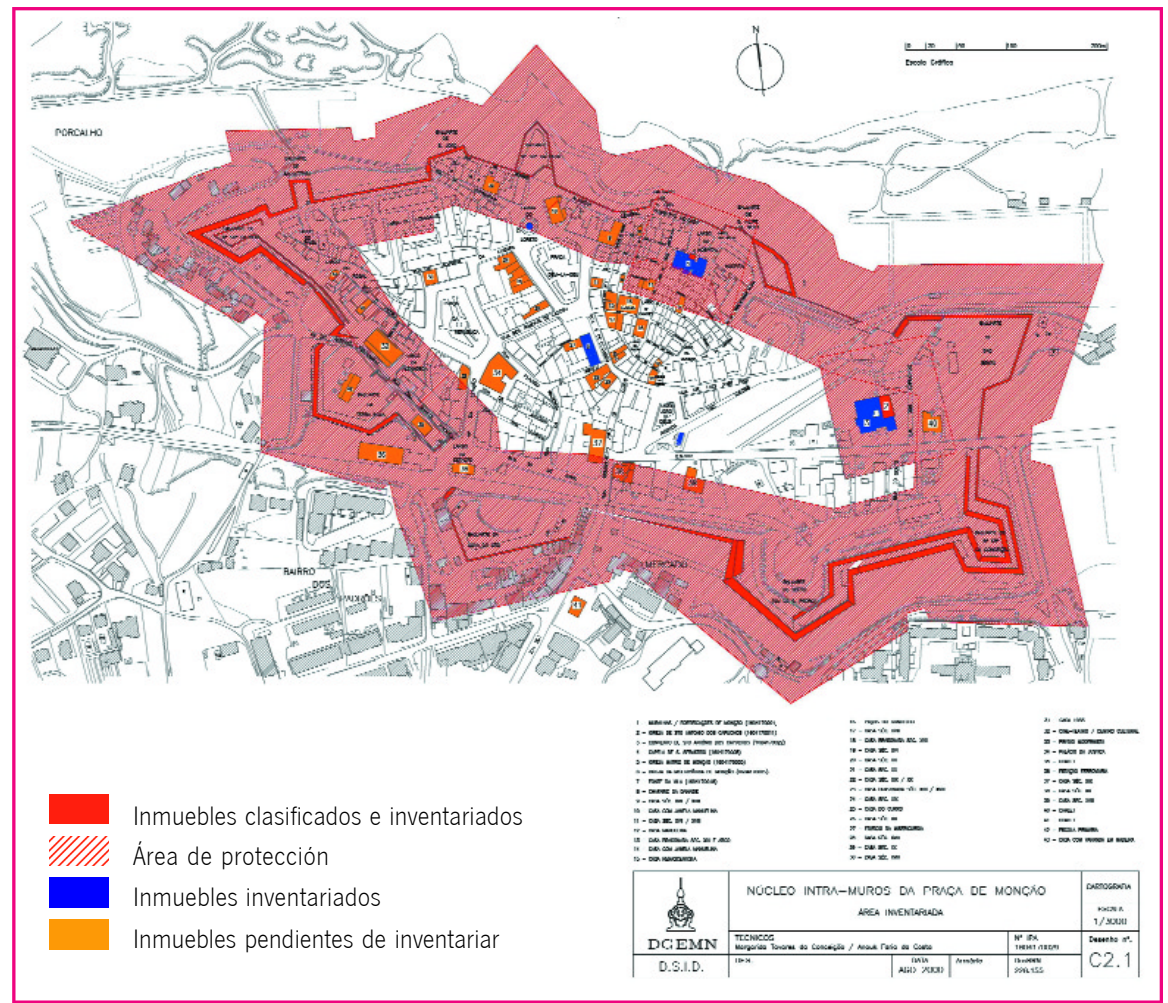

Monçao. Area inventariada

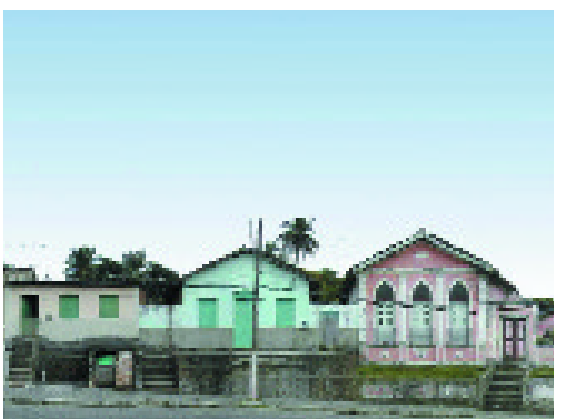

Marechal Deodoro. Calle Ladislau Neto

> Servir de base al desarrollo del proyecto de intervención, reduciendo, con enormes beneficios, el recurso al diseño

> Cuantificar los recursos financieros necesarios para la rehabilitación de las fachadas

$>$ Actualizar las diferentes bases de datos que constituyen el sistema de información.

La producción interactiva de cartas temáticas, relacionando atributos (base de datos) con datos geométricos (poligonos) se convierte en un valioso auxiliar de lectura de la información facilitando la gestión y el proceso participativo de la comunidad en la exploración de sinergias entre el tejido his- tórico, su conservación y la regeneración sostenible de las ciudades.

Considerando el potencial informativo del IPA y, gracias al desarrollo producido en la Tecnologías de la Información, pusimos a disposición una parte significativa de este sistema en la Web (www.monumentos.pt ) facilitando al público la utilización interactiva de este recurso para el desarrollo de programas educativos, turísticos y de investigación.

Concebido como un proceso multidisciplinar de transformación de datos en información, de información en conocimiento, de conocimiento en nuevos datos, el Inventario de Conjuntos Urbanos permite analizar, interpretar, comprender y proporcionar la apropiación del patrimonio por parte de la comunidad a la que pertenece, como recurso generador de riqueza e identidad.

\section{Margarida Alçada}

Directora del Servicio de Inventario

y Difusión

Dirección General de Edificios

y Monumentos Nacionales de Portugal 